\title{
A RESULT ON THE WEIL ZETA FUNCTION
}

\author{
BY \\ SAUL LUBKIN
}

1. Results. We recall Weil's conjectures [4] about the zeta function of a complete, nonsingular algebraic variety $X$ over the field of $q$ elements, $q$ a prime power. We assume that $X$ is projective and that $X$ admits a projective lifting back to characteristic zero. Let $N_{v}$ be the number of points of $X$ rational over the field $k_{v}$, where $k_{v}$ is the extension of $k$ of degree $v, v \geqq 1$.

1 (LEFSCHETZ THEOREM). There exists a doubly indexed sequence

$$
\left(\alpha_{h i}\right)_{1 \leqq i} \leqq_{h} ; 0 \leqq n \leqq 2 n
$$

of algebraic integers, where $n$ is the dimension of $X$ and $\left(\beta_{n}\right)_{0 \leqq n \leqq 2 n}$ are the Betti numbers of any lifting of $X$ to characteristic zero such that

$$
N_{v}=\sum_{1 \leqq i \leqq \beta_{n} ; 0 \leqq h \leqq 2 n}(-1)^{h} \alpha_{h i}^{v} .
$$

2 (FUNCTIONAL EQUATION). $0 \leqq h \leqq 2 n$ implies that the sequences

$$
\left(q^{n} / \alpha_{h, 1}, \ldots, q^{n} / \alpha_{h, \beta_{h}}\right) \text { and }\left(\alpha_{2 n-h, 1}, \ldots, \alpha_{2 n-h, \beta_{2 n-h}}\right)
$$

are permutations of each other.

3 (RIEMANN HYYPOTHESIS). $\left|\alpha_{h i}\right|=q^{h / 2}, 1 \leqq i \leqq \beta_{h}, 0 \leqq h \leqq 2 n$.

In addition it was later conjectured that

4. If $P_{h}=\prod_{i=1}^{\beta_{h}}\left(1-\alpha_{h i} T\right), 0 \leqq h \leqq 2 n$, then the coefficients of the polynomials $P_{h}$ are rational integers.

Conjectures 1 and 2 are now known. (See [1] and [2] for two different proofs.) Conjectures 3 and 4 are still unknown. (Under the assumption of conjecture 3 for the usual absolute value on the algebraic numbers, conjecture 4 is equivalent to the assertion that, for every absolute value on the algebraic numbers extending the usual absolute value on the rational numbers, conjecture 3 holds.) In this paper I prove a previously unknown result that would follow if both 3 and 4 were known, but that is not a consequence of either 3 or 4 alone. The result is:

5. If $0 \leqq h \leqq 2 n$ then the sequences $\left(q^{h} / \alpha_{h, 1}, \ldots, q^{h} / \alpha_{h, \beta_{h}}\right)$ and $\left(\alpha_{h, 1}, \ldots, \alpha_{h, \beta_{h}}\right)$ coincide up to permutation.

Another way of stating 5 is: If the algebraic integer $\alpha$ occurs $m$ times in the sequence $\left(\alpha_{h, 1}, \ldots, \alpha_{h, \beta_{n}}\right)$ then the algebraic integer $q^{h} / \alpha$ likewise occurs $m$ times.

(To see that 3 and 4 would imply 5 note that 3 is equivalent to the assertion $\bar{\alpha}_{h i}=q^{h} / \alpha_{h i} .4$ asserts that the coefficients of $P_{h}$ are rational and in particular real.

Presented to the Society, January 24, 1967; received by the editors November 7, 1966 and, in revised form, October 24, 1967. 
Hence complex conjugation: $\alpha_{h i} \rightarrow \bar{\alpha}_{h i}$ defines a permutation of the sequence $\left(\alpha_{h i}\right)_{1 \leqq i \leqq \beta_{h}}$. But by $3 \bar{\alpha}_{h i}=q^{h} / \alpha_{h i}$. This proves 5.)

Note that for $h=n 5$ is a special case of the functional equation 2 . 2 and 5 imply

$5^{\prime}$. If $0 \leqq h \leqq 2 n$ then the sequences

$$
\left(q^{n-h} \alpha_{h, 1}, \ldots, q^{n-h} \alpha_{h, \beta_{h}}\right) \text { and }\left(\alpha_{2 n-h, 1}, \ldots, \alpha_{2 n-h, \beta_{2 n-h}}\right)
$$

are permutations of each other.

2, 5 and $5^{\prime}$ are such that any two imply the third. Since 2 is known ([1], [2]) and we prove 5 in this paper all three statements hold.

2, 5 and $5^{\prime}$ can be written in terms of the Weil polynomials $P_{h}$ :

2. $P_{2 n-h}(T)= \pm q^{\beta_{h}(n-(h / 2))} T^{\beta_{h}} P_{h}\left(1 / q^{n} T\right)$,

5. $P_{h}(T)= \pm q^{h \beta_{h} / 2} T^{\beta_{h}} P_{h}\left(1 / q^{h} T\right)$, and

5'. $P_{2 n-h}(T)=P_{h}\left(q^{n-h} T\right), \quad 0 \leqq h \leqq 2 n$.

Another way of stating 5 is:

5. The polynomial $P_{h}\left(q^{-h / 2} T\right)$ is either symmetric or antisymmetric, $0 \leqq h \leqq 2 n$.

$5^{\prime}$ written in terms of the Weil polynomials is particularly simple. Since 2 is well known ([1], [2]) the new result 5 proved in this paper is equivalent to 5 '.

I also note (and leave it as an exercise to the reader) that if the "Riemann hypothesis" were known then the new result 5 would be equivalent to the assertion that the coefficients of the Weil polynomials $P_{h}$ are real, $0 \leqq h \leqq 2 n$, thus implying a portion of 4 . Also the new result 5 does give some information about the Riemann hypothesis 3. Namely it implies that those $\alpha_{h i}$ such that $\left|\alpha_{h i}\right| \neq q^{h / 2}$ occur in pairs $\alpha_{h i}, \alpha_{h i^{\prime}}$ where $\alpha_{h i} \cdot \alpha_{h i}=q^{h / 2}$.

5 is also equivalent to the assertion that each of the Weil polynomials $P_{h}$ factors, uniquely up to order, into a product of !inear factors: $\left(1-q^{h / 2} T\right),\left(1+q^{h / 2} T\right)$ and quadratic factors: $1+u_{j} T+q^{n} T^{2}$ where the $u_{j}$ are algebraic integers $\neq \pm 2 q^{n / 2}$. The Riemann hypothesis is equivalent to the assertion that the $u_{j}$ are all real.

Our proof of 5 is elementary. Cohomology is used; either of our well-known cohomology theories ([1], [2]) suffices. The main new idea is to apply a very simple and, probably, previously unobserved result about the characteristic polynomial of a linear transformation of a finite dimensional vector space that preserves some nondegenerate inner product (2.1).

2. Nondegenerate inner products on a finite dimensional vector space. Let $V$ be a finite dimensional vector space over a field $k$. An inner product on $V$ is a linear transformation from $V \otimes_{k} V$ into $k$, the image of the element $v \otimes w$ being written as $v \cdot w, v, w \in V$. A linear transformation $f: V \rightarrow W$ of finite dimensional vector spaces with inner products over $k$ preserves inner products if $v, w \in V$ implies $f(v) \cdot f(w)=v \cdot w$.

An inner product on the finite dimensional vector space $V$ is nondegenerate if $0 \neq v \in V$ implies there exists $w \in V$ such that $v \cdot w \neq 0$. (Notice that we do not require that $v \neq 0$ implies $v \cdot v \neq 0$-such an inner product is definite-, that $v, w \in V$ 
implies $v \cdot w=w \cdot v$-such an inner product is symmetric-or that $v, w \in V$ implies $v \cdot w=-w \cdot v$-such an inner product is antisymmetric.)

Let $P(X)=a_{n} X^{n}+\cdots+a_{1} X+a_{0}, a_{n} \neq 0$, be a polynomial of degree $n$ over a field. Then the reverse of $P$ is the polynomial $a_{0} X^{n}+a_{1} X^{n-1}+\cdots+a_{n-1} X+a_{n}$. The polynomial is symmetric (respectively: antisymmetric) if it coincides with its reverse (respectively: with the negative of its reverse).

THEOREM 1. Let $V$ be a finite dimensional vector space over a field together with a nondegenerate inner product. Let $f: V \rightarrow V$ be a linear transformation that preserves the inner product. Then the characteristic polynomial of $f$ is either symmetric or antisymmetric.

Proof. Let $f^{t}: V \rightarrow V$ be the transpose of $f$ with respect to the given inner product. Then $f^{t}$ is the unique linear transformation such that

(1) $f^{t}(v) \cdot w=v \cdot f(w), v, w \in V$.

If $\left(e_{i}\right)$ is a basis of $V$ and if $\left(e_{i}^{\prime}\right)$ is the dual basis of $\left(e_{i}\right)$ with respect to the given inner product then the matrix of $f^{t}$ with respect to $\left(e_{i}^{\prime}\right)$ is the transpose of the matrix of $f$ with respect to $\left(e_{i}\right)$. Hence $f$ and $f^{t}$ have the same characteristic polynomial.

Since $f$ preserves the inner product we have $f^{t}(f(v)) \cdot w=f(v) \cdot f(w)=v \cdot w$, $v, w \in V$. Hence $f^{t}(f(v))=v, v \in V$,

(2) $f^{t} \circ f=$ identity.

Hence $f$ is an automorphism of $V$. Since $f^{t}$ and $f$ have the same characteristic polynomial they have the same determinant. Taking the determinant of (2) gives

(3) $\operatorname{det} f= \pm 1$.,

Considering the definition of the characteristic polynomial as det (identity $X-f$ ) and using the fact that $\operatorname{det}(f)= \pm 1$ we see that the characteristic polynomial of $f^{-1}$ is \pm the reverse of the characteristic polynomial of $f$. By (2) $f^{-1}=f^{t}$ which has the same characteristic polynomial as $f$. Hence the characteristic polynomial of $f$ is either symmetric or antisymmetric.

COROllaRY 1.1. Let $V$ be a finite dimensional vector space over a field and let $f: V \rightarrow V$ be a linear transformation that preserves some nondegenerate inner product. Let $\left(\alpha_{1}, \ldots, \alpha_{n}\right)$ be the sequence of eigenvalues with multiplicities of $f$. Then the sequences $\left(\alpha_{1}, \ldots, \alpha_{n}\right)$ and $\left(\alpha_{1}^{-1}, \ldots, \alpha_{n}^{-1}\right)$ coincide up to permutation.

Proof. A polynomial is either symmetric or antisymmetric if and only if the mapping: $\alpha \rightarrow \alpha^{-1}$ is a bijection of the roots preserving multiplicities. Hence the corollary is equivalent to the theorem.

3. Proof of 1.5. The notations being as in $\S 1$ let $\mathcal{O}$ be a complete discrete valuation ring with quotient field of characteristic zero such that $X$ admits a projective lifting $X$ over $\mathcal{O}$. Fix a complex imbedding: $\mathcal{O} \subset C$ and let $X_{C}$ be the projective nonsingular complex algebraic variety

$$
X_{C}=X \times_{\operatorname{Spec}(\mathcal{O})} \operatorname{Spec}(C) .
$$


Let $K$ be either the ring of $q^{\prime}$-adic integers, $q^{\prime}$ a rational prime unequal to the characteristic of $k$, or the quotient field of $\mathcal{O}$. Let $H^{*}(X, K)$ be either the $q^{\prime}$-adic [1] or the $K$-adic [2] cohomology of $X$ respectively. If $K=\hat{Z}_{q^{\prime}}$ then let $K \subset C$ be a complex imbedding of the ring $K$. In either case let $H^{*}(X, C)=H^{*}(X, K) \otimes_{K} C$. Then the ring $H^{*}(X, C)$ is canonically isomorphic to the classical complex cohomology algebra $H^{*}\left(X_{C}, C\right)$ of the complex algebraic variety $X_{C}$. Identify these two rings.

Let $u \in H^{2}\left(X_{C}, C\right)$ denote the "Kähler class" ([5], see also [3])-the class. of a generic hyperplane section of $X_{C}$. Then $u \in H^{2}(X, C)=H^{2}(X, K) \otimes_{K} C$ is the canonical class of a generic hyperplane section $H$ of $X$ ([1], [2]). Hence if $f^{*}=$ $\left(f^{h}\right)_{0 \leqq h \leqq 2 n}$ denotes the maps induced by the Frobenius ([1], [2]) on the cohomology groups $H^{*}(X, \boldsymbol{C})=\left(H^{n}(X, C)\right)_{0 \leqq n \leqq 2 n}$ then

(1) $f^{2}(u)=q \cdot u$.

$f^{*}: H^{*}(X, C) \rightarrow H^{*}(X, C)$ preserves cup products. Hence if we define

(2) $g^{h}=q^{-h / 2} \cdot f^{h}, \quad 0 \leqq h \leqq 2 n$,

then $g^{*}: H^{*}(X, C) \rightarrow H^{*}(X, C)$ preserves cup products and $g^{2}(u)=u$.

By the functional equation 1.2 , to prove 1.5 it suffices to consider the case $0 \leqq h \leqq n$.

If $0 \leqq h \leqq n$ and $v, w \in H^{h}\left(X_{C}, C\right)$ then define $v \cdot w=u^{n-h} \cup v \cup w$. Then [5] the assignment $(v, w) \rightarrow v \cdot w$ is a nondegenerate inner product on the finite dimensional vector space $H^{h}\left(X_{C}, C\right)$. Since $g^{*}$ preserves cup products and $g^{2}(u)^{\prime}=u$ it follows that $g^{h}$ preserves this nondegenerate inner product. Hence by 2.1 .1 if $\left(a_{h, i}\right)_{1 \leqq i \leqq b_{h}}$ are the eigenvalues of $g^{h}$ with the appropriate multiplicities then

(3) The sequences $\left(a_{h, i}^{-1}\right)_{1 \leqq i \leqq b_{h}}$ and $\left(a_{h, i}\right)_{1 \leqq i \leqq b_{h}}$ are permutations of each other.

The sequence $\left(\alpha_{h, i}\right)_{1 \leqq i \leqq \beta_{h}}$ of $\S 1$ is the sequence of eigenvalues with multiplicities of the linear transformation $f^{h}: H^{h}(X, C) \rightarrow H^{h}(X, C)$, modified in a manner similar to the Remark, p. 253 of [2] to make all the $\alpha_{h, i}$ algebraic integers. Hence by (2)

(4) The sequences $\left(\alpha_{h, i}\right)_{1 \leqq i \leqq \beta_{h}}$ and $\left(q^{h / 2} a_{h, i}\right)_{1 \leqq i \leqq b_{h}}$ coincide up to permutation.

(3) and (4) imply 1.5 .

\section{BIBLIOGRAPHY}

1. S. Lubkin, On a conjecture of André Weil, Amer. J. Math. 89 (1967), 443-548.

2. - A p-adic proof of Weil's conjectures, Ann. of Math. (2) 87 (1968), 105-255.

3. J.-P. Serre, Analogues kählériens de certaines conjectures de Weil, Ann. of Math. (2) 71 (1960), 392-394.

4. A. Weil, Number of solutions of equations over finite fields, Bull. Amer. Math. Soc. 55 (1949), 497-508.

5. - Introduction à l'étude des variétés kählériennes, Actualités Sci. Indust., No. 1267, Hermann, Paris, 1958; Russian transl., IL, Moscow, 1961.

UNIVERSity OF CALIFORNIA,

Berkeley, CALIFornia 